\title{
An Energy Efficient Approach for Clustering in WSN using Fuzzy Logic
}

\author{
Ashutosh Kumar Singh, Sandeep Goutele, S.Verma and N. Purohit \\ Indian Institute of Information Technology, Allahabad \\ Allahabad, India
}

\begin{abstract}
Lifetime augmentation has always been an interesting subject of vital significance in wireless sensor networks. Excess extent of energy is dissipated during data transmission to the base station (sink) from normal sensor nodes. Clustering is an efficacious way of augmenting the life span by optimizing (reducing) the energy dissipation of WSNs. Many numbers of researchers have employed fuzzy logic based solution for election of cluster- head in the earlier past. In our proposed scheme two strong parameters; energy and centrality have been considered for cluster head decision. In this work we assumed both the parameters simultaneously to elect cluster head which makes this approach energy efficient and more feasible. Simulation results are contrasted with the former approaches of cluster-head election. Simulation results exhibits that the proposed scheme minimizes energy consumption and thereby enhances life span of wireless sensor network by a significant amount.
\end{abstract}

\section{Keywords}

fuzzy logic; wireless sensor network; cluster-head election

\section{INTRODUCTION}

A wireless sensor network (WSN) entail of spatially distributed self-sufficient sensors to monitor physical or conservational requirements, such as pressure, sound, vibration, temperature, motion or pollutants and to collaboratively pass their data to a main location(base station or sink) through the network. In present most of the latest networks are bi-directional, enabling to cope up with the activity of the sensors too. Military applications like battlefield reconnaissance is the main inspiration for wireless sensor networks development; recently this type of distributed networks are adopted in most of remote monitoring applications and industrial measurements application like machine condition monitoring, industrial process monitoring, structural health monitoring, air/climate, water/soil, indoor monitoring and so on

A WSN is entail of "sensors" which we say nodes - deployed from some sensor nodes to numerous hundreds or even thousands numbers of nodes, where every sensor node is associated by radio link to one or sometimes several other sensors nodes. Each such sensor network node comprises of typically several parts: an electronic circuit to interacting with other sensors nodes and an external energy source (sensors are battery operated), a radio transceiver with an association to an external antenna or may have an embedded internal antenna, a microcontroller and typically an embedded form of energy source or a battery.

Broad range of applications like disaster management (seismically sensitive zone), security and military have stimulated the importance in WSN during few past years. Sensors nodes are characteristically proficient of wireless communication and are considerably obliged in the amount of existing resources such as energy (power), storage (memory) and computation. These obligate make the deployment and operation of WSN significantly distinct from existing wireless networks, and demand the development of resource aware protocols and supervision techniques.

The categorization of WSNs is depends on their applications which classified it's into following types- i) event driven WSN ii) time driven WSN iii) an amalgam (hybrid) of the above two categorization. Different WSN's applications have different requirements.

\subsection{Clustering approach}

In the cluster-based approach, solely some of sensor nodes in particular WSN are permitted to transmit sensed data towards the base station. The primary issue is that, this allows sensor nodes to sense and transmit the sensed information (in data packets form) to the cluster-heads directly, instead of routing through its immediate neighbors. Also, since energy dissipation during communication is proportionate to the square of distance to the base station from the sending sensor node, having all nodes to transmit its sensed data individually to the base station, exhausts the energy of energy of each node drastically and hence the lifetime operation of the network gets significantly reduced. As a subsequent result it doesn't serve the objective with which WSNs are designed for, namely the network should be long lasting, significant and computable for a long-lasting period of time.

\subsection{Problem in cluster-head selection}

Clustering is useful in WSN to decrease the total energy loss inside the network by permitting only some of the selected nodes to take contribution in the data transmission directly to destination (base station). However it helps to save the transmission bandwidth too and thus increase better resource utilization, and improve power control.

LEACH is a popular current approach for cluster head selection and prepared the basis for many approaches. Algorithm like LEACH uses probabilistic approach to select cluster-head 
schematically. But this method use only local information to take decision of cluster-head so using only local information has its own limitations. Since each node probabilistically elects whether or not to become cluster-head, there might be cases when two cluster-heads are selected in the closed proximity of each other. In reality considering only one factor like energy, is not suitable to elect the cluster-head properly. This is because other conditions like centrality of nodes corresponding to the entire cluster, gives an amount of the entire dissipation during transmission for all nodes too. The more central the node to a cluster the more is the energy efficiency for other nodes to transmit through that selected node.

Most of the WSNs applications require long lifetime of the networks. Many times WSNs are deployed in an area (application dependent) where it is challenging to replace the nodes quite often. There is limited amount of sensor's energy for each node for communication with command node. Sensor nodes can be networked into well-defined clusters. Nodes in a cluster can directly communicate with cluster-head. Hence in clustered network overall energy consumption is highly reduced. Each cluster has a cluster-head like a gateway which is high energy node. The cluster-head is responsible for communication among nodes in a cluster.

\subsection{Fuzzy Logic Control}

This model comprises of four components fuzzy inference engine, fuzzy rules, fuzzifier, and a defuzzifier. As matlab simulator provides the most frequently used Mamdani Method in matlab is a fuzzy inference technique, owing to its simplicity and manipulability we have applied it in simulation. The four steps processes are:

- Fuzzification of the input parameters total dissipated energy and node centrality. Now to resolve the level to which the inputs are belonging to the appropriate fuzzy sets or rule the inputs are analyzed.

- Rule evaluation -fuzzified inputs (energy and node centrality) are applied to the originator of fuzzy inference rules. Then it is applied to subsequent membership function (Table 1).

- Resemblance of the fuzzy inference rule outputs -this is the technique of assimilation of the outputs of all fuzzy inference rules.

- Defuzzification - the resembled output fuzzy set chance is applied as input for the defuzzification technique and the output of defuzzification process is a single sharp and concise (crisp) number.

It calculates the point (result) where a vertical line would segment the resembled set chance into two identical masses during defuzzification.

\section{RELATED WORKS}

A typical Wireless Sensor Network's architecture is expressed by following Figure 1. All the sensor nodes in one cluster send their sensed information to elected respective cluster-heads; the cluster-head in turn then integrate or aggregate and then compress the sensed data and transmit directly it to the base station.

Following assumptions are taken for WSN:

- The base station (sink) is stationary and it is established to a stipulated distance from the normal sensor nodes.

- The nodes are homogeneous.
- All the nodes in WSN are energy constrained.

- Propagation channel is symmetric.

- Sink is responsible for the cluster-head election.

- Nodes have location information means nodes are gps equipped.

- Nodes have little or no mobility.

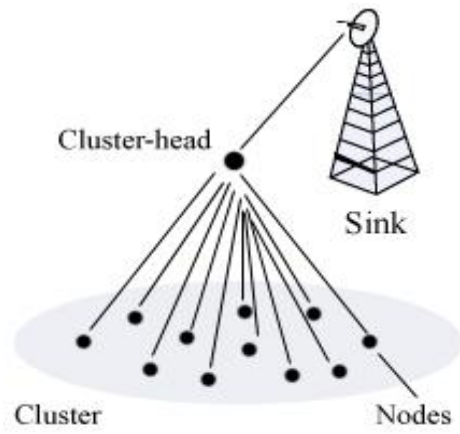

Figure 1 WSN architecture

Prolonging the life of a WSN is a past research topic itself; in spite of that for extending the lifetime of WSN many number of researches find it as an interesting research area. A number of algorithms, strategies and approaches have been formulated earlier and mostly of them are based on routing algorithms to optimize (reduce) the energy dissipation of nodes in WSN. In [1], authors have given a scheme for clustering in which the cluster-head election is depends on selecting a sensor node which has maximum residual (remaining) energy (after all the communication happened in recent round) or the distance to the centroid (based on deployment) of a particular cluster, and the normal sensor node which one is closest elected as the cluster-head. In [2] the suggested solution is based on fuzzy logic too; the three important parameters residual energy, density and node centrality is assumed to increases the lifetime of network as compare to LEACH. In [3] a new cluster-head election scheme "Cluster Head Election using Fuzzy logic" (CHEF) is introduces. In which the collecting and calculating overheads is reduced by using fuzzy logic by this finally the life of WSN can be extended. CHEF is about $22.7 \%$ more efficient than LEACH.

In [4] the authors propose two fuzzy-based systems for cluster head selection in sensor networks to deal with this problem, when a sensor with appropriate battery and computational power detects signals of interest, it is decided to act as a $\mathrm{CH}$. That was a simple method, because no explicit $\mathrm{CH}$ election process is obliged and, therefore no extreme message exchanges are obliged. Moreover, selection of $\mathrm{CHs}$ by this way is challenging in different environments having different characteristics such as error rate, SNR, throughput and so on. In [11] the author suggested a hybrid methodology for clustering in WSNs HEED is the main motivation in this approach. This suggested approach is very useful for the applications that oblige scalability and prolonged network lifetime. In this approach the clustering won't be performed until at least one of the $\mathrm{CHs}$ consume a predefine part of its energy, that's why if the clustering decision is taken at the beginning (after the first setup phase) of each round it imposes lots of overhead on the network.

In [5] author introduces a new protocol for WSN, which uses the hierarchical clustering approach and controls data transmission by defining range of data transmission. Because of 
different cluster-head election algorithms, this protocol can be followed in both distributed and centralized way. Both of them are proficient in terms of durability of network and energy dissipation.

As the LEACH is the most famous routing approach. Moreover, in LEACH, in every $1 / \mathrm{p}$ rounds, when a node has been decided as a cluster-head, 0 will be set as its threshold, which shows the node has dropped its chance to take participation in next cluster-head election round, even if it still has obliged residual energy present. So in [6] a novel clusterhead election algorithm is suggested. In this scheme in place of changing the threshold value to 0 , it is adjusts gradually as per the roles the nodes have played in the earlier election round, so more other sensor nodes could get the chance to take part in cluster-head election. So the proposed approach performs LEACH in network by a typical amount of $30 \%$ approximately

In [7] a two-level fuzzy logic is adopted to evaluate the probability of sensor node to elect as a cluster head. In the first level (Local Level), node's energy and number of neighbors are used as parameter to decide node as cluster-head. In the second level (Global Level), node's overall cooperation is considered to the whole network with three fuzzy parameters. These parameters are centrality, proximity to base station and distance between cluster heads. This proposed approach consumes less energy and improve the life span of network about $54 \%$ compared with other previous approaches.

In [13] author proposes a scheme to resolve the bottle neck happening in cluster-based sensor networks. For networks a Transmission tuning algorithm is proposed it depends on the modeling of the further overhead on the sensor nodes that have straight communication with the sink. The proposed idea can accomplish a longer overall life span of a sensor network about $35 \%$ longer than traditional HEED protocol.

In the proposed scheme two important parameters are considered as the cluster-head election constraint these are:

Node Energy: overall energy level is obtainable in each sensor node, expressed by the fuzzy variable energy, the energy value is scaled while representing in the fuzzy set,

Node Centrality: how central the node is to the cluster is classified by the centrality property which is expressed by the fuzzy variable centrality. The centrality value is scaled while representing in the fuzzy set.

\section{FUZZY LOGIC SCHEME}

We have considered two inputs for the intended fuzzy logic inference mechanism. These are (i) residual energy of node (ii) Centrality of node. In the first place all input data are fuzzified in a number of fuzzy sets. A node with lesser energy is given high priority. Also nodes distant from the base station are given high priority. Table 1 is rule base for this inference mechanism. Figure 2 shows fuzzy membership function for input variable residual energy. Figure 3 shows fuzzy membership function for input variable centrality from the base station. Three values have been decided for the residual energy input variable these are (i) Low (ii) Medium (iii) High. Three values are again chosen for centrality variable these are (i) Close (ii) Adequate (iii) Far. Figure 4 shows fuzzy membership function for output "Probability". Nine subsets have been chosen for output variable these are (i) v-Small (ii) Small (iii) r-Small (iv) m-Small (v) Medium (vi) m-Large (vii) r-Rather (viii) Large (ix) v-Large. In this scheme after each round the remaining energy of nodes and centrality of nodes are analyzed. If the residual energy is low and value of centrality is closed then according to fuzzy sets the output probability is r-Small. If for residual energy value high the value of centrality is close then the output probability will be v-Large and so on.

Therefore fuzzy logic is a simple and useful tool for optimal choice making in such cases.

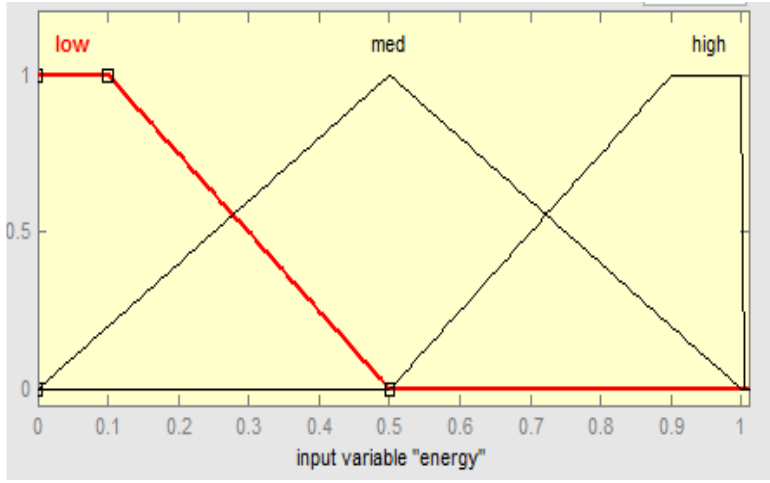

Figure 2 Fuzzy membership functions for "Energy"

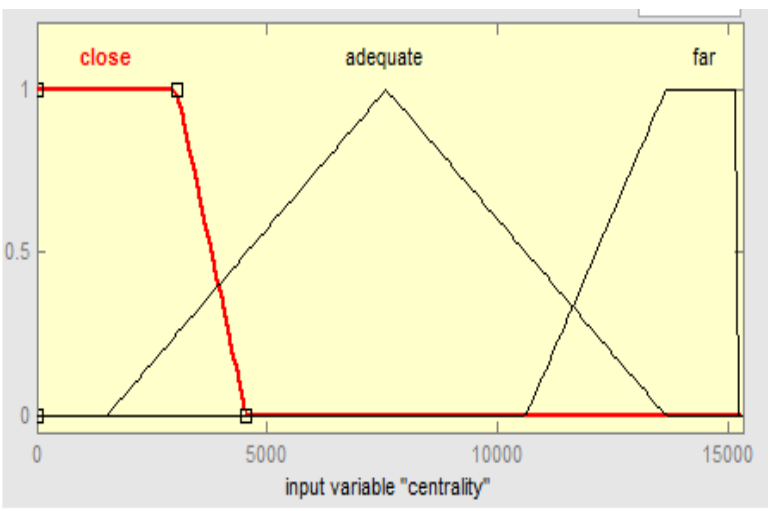

Figure 3 Fuzzy membership functions for "Centrality"

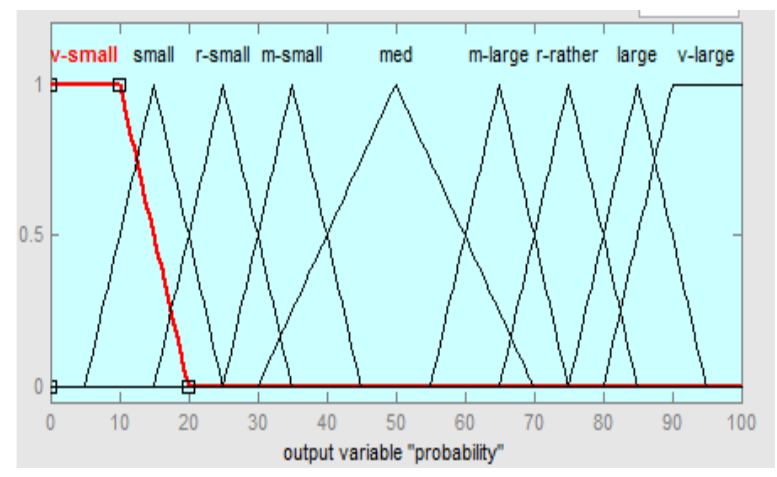

Figure 4 Fuzzy membership functions for output Table1. Fuzzy Rule Base Table

\begin{tabular}{|c|c|c|}
\hline Energy & Centrality & Probability \\
\hline Low & Close & r-Small \\
\hline Low & Adequate & Small \\
\hline
\end{tabular}




\begin{tabular}{|c|c|c|} 
Low & Far & v-Small \\
\hline Med & Close & m-Large \\
\hline Med & Adequate & Medium \\
\hline Med & Far & m-Small \\
\hline High & Close & v-Large \\
\hline High & Adequate & Large \\
\hline High & Far & r-Rather \\
\hline
\end{tabular}

\section{SIMULATION RESULTS}

We have selected MATLAB software for the simulation work. We have performed four simulations with varying number of sensor nodes. In these simulations we have taken initial energy of each sensor nodes is $1 \mathrm{~J}, \mathrm{E}_{\text {elect }}=50 \mathrm{~nJ} / \mathrm{bit}, \varepsilon_{\mathrm{fs}}=10 \mathrm{pJ} / \mathrm{bit} / \mathrm{m}^{2}$ and $\varepsilon_{\mathrm{mp}}=1.3 \times 10^{-3} \mathrm{pJ} / \mathrm{bit} / \mathrm{m}^{2}$ for our first simulation. The simulation graphs shows the comparison of lifetimes of WSN after applying leach based clustering (in blue line), and then applying residual energy-centrality fuzzy based clustering (in red line).

In our first simulation we took $n=200$ nodes, figure 5 shows the simulation result WSN's lifetime is measured about 1020 rounds when leach based clustering is applied and the life time is extended to 1868 rounds when energy-centrality based clustering is applied.

For our second simulation we took $n=200$ sensor nodes figure 6 shows the simulation result WSN's lifetime is measured about 1051 rounds when leach based clustering is applied and the life time is extended to 1878 rounds when energy-centrality based clustering is applied.

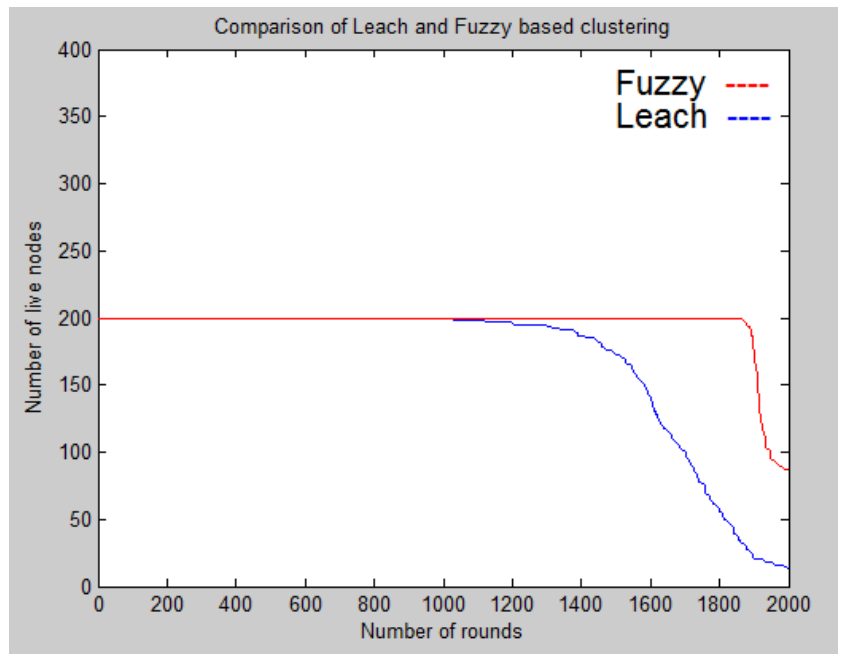

Figure 5 Comparison of Lifetimes of wsn with leach based clustering and Energy- Centrality fuzzy based clustering.

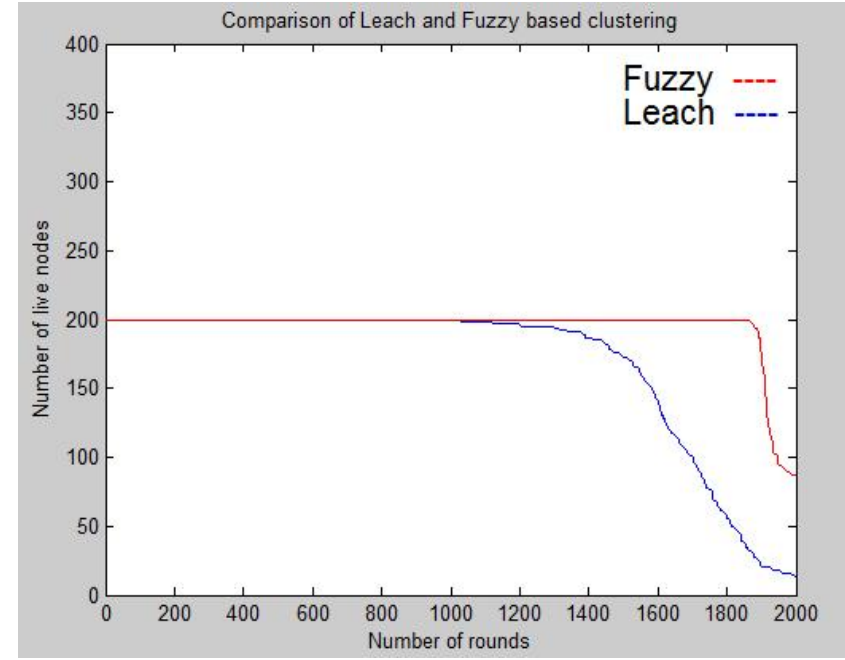

Figure 6 Simulation result with $\mathbf{n}=\mathbf{2 0 0}$ nodes

For subsequent simulation we took $n=250$ sensor nodes figure 7 shows the simulation result WSN's lifetime is measured about 1072 rounds when leach based clustering is applied and the life time is extended to 1482 rounds when energy-centrality based clustering is applied.

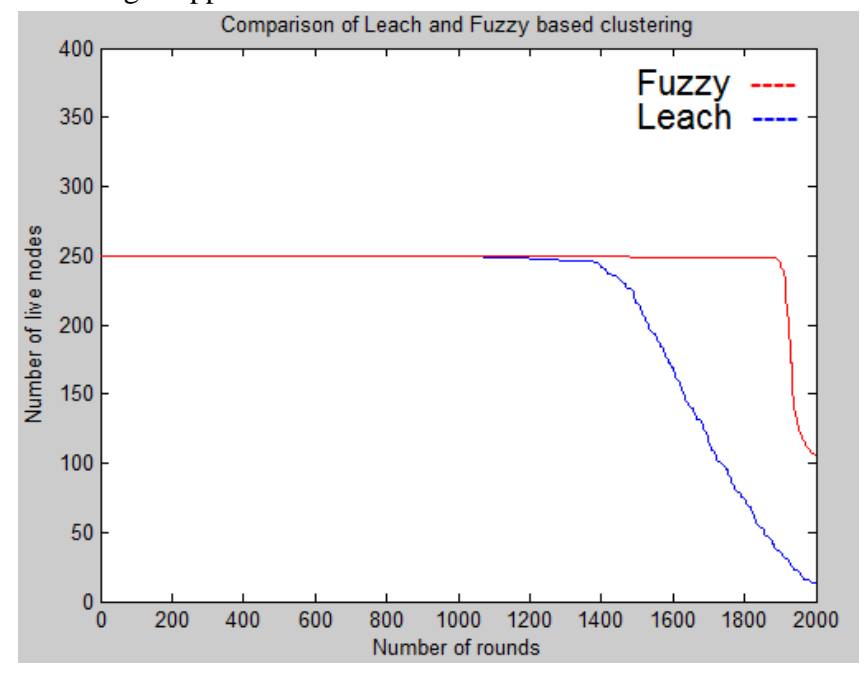

Figure 7 Simulation result with $\mathbf{n}=\mathbf{2 5 0}$ nodes

For our last simulation we took $\mathrm{n}=500$ sensor nodes the simulation result is shown in figure 8 which shows the network's life time is about 1117 rounds when leach based clustering is applied to the wireless sensor network and 1418 rounds when energy-centrality based clustering is applied. 


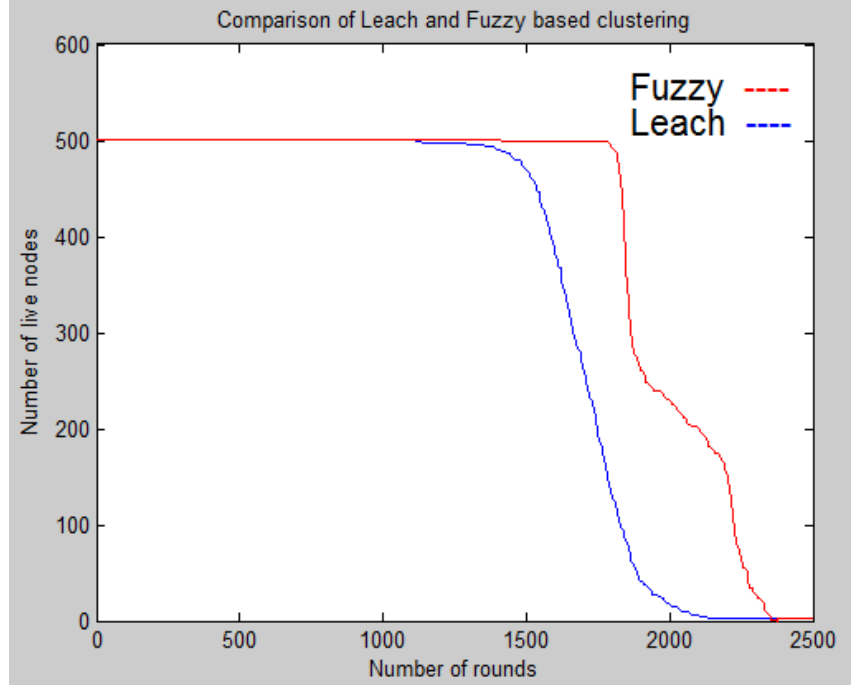

Figure 7 Simulation result with $\mathbf{n}=500$ nodes

\section{CONCLUSION}

We have proposed a unique strategy for the cluster-head election in wireless sensor network by taking two important parameters; residual energy of the nodes and the centrality of the node using fuzzy logic and this approach has been successful in prolonging the lifetime by a significant amount as compare to very basic LEACH approach. According to simulation results we find that this fuzzy based scheme enhances the network lifetime near about $70 \%$ as compare to probabilistic LEACH approach.

\section{REFERENCES}

[1] Q. Liang, "Clusterhead election for mobile ad hoc wireless network," in Proc. 14th IEEE International Symposium on Personal, Indoor and Mobile Radio Communications, (PIMRC) , pp. 1623 -1628, Sept. 2003.

[2] Indranil Gupta, Denis Riordan and Srinivas Sampalli, "Cluster-head Election using Fuzzy Logic for Wireless Sensor Networks", the 3rd Annual Communication Networks and Services Research Conference (CNSR'05), pp. 255 - 260, 2005.

[3] Jong-Myoung Kim, Seon-Ho Park, Young-Ju Han and TaiMyoung Chung, "CHEF: Cluster Head Election mechanism using Fuzzy logic in Wireless Sensor Networks", Advanced Communication Technology, 2008. ICACT 2008. 10th International Conference, pp. 654 - 659, vol. 1, 2008.

[4] Junpei Annoa, Leonard Barollib, Arjan Durresic, Fatos Xhafad and Akio Koyamae, "Performance evaluation of two fuzzy-based cluster head selection systems for wireless sensor networks", Network-Based Information Systems (NBiS), 2010 13th International Conference pp. 55-61, 2010

[5] Ning Sun, Nai-bin Su and Sang-ho Lee, "A Lifetime Extended Routing Protocol Based on Data Threshold in Wireless Sensor Networks", 10th IEEE International Conference on Computer and Information Technology (CIT 2010), pp. 743-748, 2010.

[6] Rong Ding, Bing Yang, Lei Yang and Jiawei Wang, "Soft Threshold Based Cluster-head Selection Algorithm for Wireless Sensor Networks", Third International Conference on Sensor Technologies and Applications, pp. 526-530, 2009 .

[7] Nasrin Abazari Torghabeh, Mohammad Reza Akbarzadeh Totonchi and Mohammad Hossein Yaghmaee Moghaddam, "Cluster Head Selection using a Two-Level Fuzzy Logic in Wireless Sensor Networks", Computer Engineering and Technology (ICCET), 2010 2nd International Conference, pp. 357-361, vol. 2, 2010.

[8] Hironori Ando, Leonard Barolli, Arjan Durresi, Fatos Xhafa, and Akio Koyama, "An Intelligent Fuzzy-based Cluster Head Selection System for Wireless Sensor Networks and Its Performance Evaluation", 13th International Conference on Network-Based Information Systems, pp. 55-61, 2010.

[9] Hironori Ando, Leonard Barolli, Arjan Durresi, Fatos Xhafa, and Akio Koyama, "An Intelligent Fuzzy-based Cluster Head Selection System for WSNs and ItsPerformance Evaluation for D3N Parameter", International Conference on Broadband, Wireless Computing, Communication and Applications, pp. 648653,2010

[10] Sudakshina Dasgupta and Paramartha Dutta, "An improved Leach approach for Head selection Strategy in a Fuzzy-C Means induced clustering of a Wireless Sensor Network", IEMCON 2011 organised by IEM in collaboration with IEEE on 5th \& 6th of Jan,2011, pp. 203-208, 2010.

[11]Xiao $\mathrm{Fu}$ and Zhenhua $\mathrm{Yu}$, "A Reliable and Efficient Clustering Algorithm for Wireless Sensor Networks Using Fuzzy Petri Nets", Wireless Communications Networking and Mobile Computing (WiCOM), 2010 6th International Conference, pp. 1-4, 2010.

[12] A. Manjeshwar; D.P. Agrawal, "TEEN : A routing protocol for enhanced efficiency in wireless sensor networks", $15^{\text {th }}$ International Parallel and Distributed Processing Symposium, pp.2009-2015, Apr 2001.

[13]N.Eshghi and A.T.Haghighat,"Energy Conservation Strategy in Clusterbased Wireless Sensor Network", IEEE ICACTE, pp.1015- $\quad 1019, \quad 2008$ 